\title{
LA VISIÓN DE LOS ESTUDIANTES \\ DE ORIGEN EXTRANJERO SOBRE FACTORES Y COMPETENCIAS \\ CLAVE PARA EL ACCESO A LA UNIVERSIDAD
}

\section{THE VISION OF IMMIGRANT STUDENTS \\ ABOUT KEY FACTORS AND SKILLS FOR ACCES TO COLLEGE}

\section{A VISÃO DOS ESTUDANTES IMIGRANTES \\ SOBRE FACTORES E HABILIDADES FUNDAMENTAIS \\ PARA ACCESO AO ENSINO SUPERIOR}

\section{Elena Cano García y Maite Fernández Ferrer}

UNIVERSIDAD DE BARCELONA, ESPAÑA

RESUMEN: Uno de los retos educativos más importantes en la actualidad es la lucha contra el abandono escolar. Un caso especialmente relevante es el de los estudiantes inmigrantes, que, en ocasiones, son un colectivo vulnerable que aún presenta menor tasas de acceso a la universidad.

Por este motivo, en el proyecto "Competencias y factores clave para el éxito educativo desde la perspectiva de estudiantes universitarios hijos/as de inmigrantes" (2009 ARAF1 00010) se ha pretendido identificar las competencias clave y los factores más relevantes que los estudiantes universitarios hijos/as de familias inmigrantes en Cataluña consideran que les han sido útiles para conseguir el éxito educativo, entendiendo éste como el hecho de llegar a la universidad.

Para la metodología del estudio se han utilizando tres fuentes diferentes de recogida de información: los relatos de vida, las narraciones audiovisuales y el cuestionario. Concretamente, para este artículo, nos referimos a los resultados analizados a través de los relatos de vida de 13 estudiantes universitarios de origen inmigrante que habían realizado toda o parte de su escolaridad básica dentro del sistema educativo español, teniendo en cuenta para su selección la edad de llegada, su nacionalidad y la variedad de universidades y estudios de procedencia.

Los resultados del estudio apoyan los hallazgos de investigaciones previas (Aja et al. 2000; Huguet y Navarro, 2006; Ferrer, 2009; OECD, 2010; Instituto de Evaluación, 2010) en tanto que los estudiantes participantes se han referido, en la dimensión personal, a la importancia de las expectativas y del soporte familiar, especialmente cuando éste se ha dado de forma conjunta con la escuela. Han destacado especialmente el papel de las madres como figura básica para estimular la constancia y la dedicación al estudio. En el ámbito escolar han valorado especialmente el papel de algún/a profesor/a de secundaria que 
resultó clave por depositar en ellos/as altas expectativas y comunicárselas explícitamente. Finalmente, respecto a las competencias, que ha sido el eje central de nuestro análisis, de los relatos de los participantes emergen, vinculadas a la competencia intrapersonal, la autoconfianza y auto eficacia como capacidades que los estudiantes consideran básicas para superar, como indican los estudios de resiliencia, contextos y condiciones adversas.

PALABRAS CLAVE: Inmigración; familia; éxito académico.

ABSTRACT: One of the most important challenges in education is working against absenteeism and school dropout. Especially relevant is the case of immigrant students who are a vulnerable group, fact that causes a high difference in results between immigrants and native students, so that only a very small percentage reaches college.

For this reason, the study "Competencies and keys for an educational success from the perspective of college students' children of immigrants" (2009 ARAF1 00010) has sought to identify the key factors and skills that college students from immigrant families in Catalonia (Spain) feel have been useful for their educational success, educational success seen as the enrolment in programs beyond compulsory education.

The study methodology has been predominantly qualitative using three different sources of data collection: life stories, audiovisual narratives and questionnaires. Specifically for this article we refer to the results analyzed through the life stories of 13 college students from immigrant backgrounds who had done all or part of their primary school in the Spanish education system, considering for their selecting the age of arrival, their nationality and the variety of universities and degrees where they were enrolled.

The results of the study support the findings of previous researches (Aja et al. 2000; Huguet \& Navarro, 2006; Ferrer, 2009; OECD, 2010; Instituto de Evaluación, 2010). Student participants have referred to the importance of family expectations and support, especially when it has been given in conjunction with the school. They especially emphasized the role of mothers as a basic figure to encourage perseverance and dedication to studies. Moreover, in the educational environment it has been particularly appreciated the role the high school teachers who in many cases were the key on having great expectations for them and communicating these explicitly. Finally, regarding the competences, which have been the focus of our analysis, the intrapersonal and the self-confidence competences are the skills that students consider basic to overcome, as indicated by studies about resilience, adverse contexts and conditions.

KEY WORDS: Immigrants; Family; Academic Achievement.

RESUMO: Um dos desafios educativos mais importantes da atualidade é a luta contra o absentismo e abandono escolar. Um grupo particularmente vulnerável neste contexto são os alunos imigrantes, verificandose uma diferença grande entre os resultados escolares de imigrantes e nativos, no sentido em que apenas uma percentagem muito reduzida destes alunos ingressa na universidade.

Neste contexto, no projeto "Competências e fatores chave para o sucesso educativo - a perspectiva de estudantes universitários filhos/as de imigrantes" (2009 ARAF1 00010), pretendeu-se identificar as competências chave e os fatores considerados mais relevantes pelos estudantes universitários filhos/as de imigrantes na Catalunha para o seu sucesso escolar, operacionalizando-se o sucesso escolar como o acesso à universidade.

A metodologia do estudo foi eminentemente qualitativa, tendo-se utilizado 3 fontes para recolha de informação: os relatos de vida, as narrativas audiovisuais e o questionário. Concretamente para este artigo referiremos os resultados analisados através dos relatos de vida de 13 estudantes universitários de origem imigrante que realizaram toda, ou parte, da sua escolaridade básica dentro no sistema educativo espanhol, tendo em conta, para a sua seleção, a idade de entrada, a sua nacionalidade e a diversidade de universidades e estudos de procedência.

[ 264 ] ELENA CANO GARCÍA Y MAITE FERNÁNDEZ FERRER 
Os resultados do estudo apoiam as conclusões de investigações anteriores (Aja et al. 2000; Huguet \& Navarro, 2006; Ferrer, 2009; OECD, 2010; Instituto de Evaluación, 2010), uma vez que os estudantes mencionaram como fatores importantes para o sucesso escolar, a dimensão pessoal e as expectativas e apoio da família, particularmente quando este apoio se verifica em paralelo também na escola. Destacaram especialmente o papel das mães como figura central no suporte e motivação para os estudos. Em contexto escolar, os sujeitos inquiridos valorizaram particularmente o papel de um professor/a do ensino secundário, como uma figura chave que lhes depositou confiança e lhes comunicou explicitamente expectativas altas. Finalmente, a respeito das competências, que foram o eixo central da nossa análise, dos relatos dos participantes no estudo emergem, associadas à competência intrapessoal, a autoconfiança e autoeficácia como capacidades que os estudantes consideram básicas para superar, tal como indicam os estudos na área da resiliência, contextos e condições adversas.

PALAVRAS-CHAVE: Imigração; família; sucesso académico.

\section{Introducción}

Desde la década de los 90, el flujo de llegada de la población extranjera a España provocó un incremento muy rápido de la participación del alumnado inmigrante en el sistema educativo. En los últimos años anteriores a la crisis económica, la presencia de alumnado extranjero en la educación obligatoria aumentó por encima del 500\%. Este fenómeno se dio especialmente en Cataluña, donde durante el curso 2008-2009 el 15,9\% de los estudiantes de educación obligatoria eran extranjeros.

El acceso a los estudios universitarios, aunque se trate de niveles no obligatorios, es un indicador de cohesión social, sobre todo, para los inmigrantes que ya llevan décadas establecidos y para segundas generaciones en el país. Por desgracia, en algunos casos, estos procesos migratorios que viven estos estudiantes han repercutido negativamente en las trayectorias académicas de los y las alumnas que han emigrado, ya sea por la ruptura que se ha producido en estos procesos educativos, por las situaciones de duelo que algunos de estos niños, niñas y jóvenes adolecentes viven, o bien por las dificultades de éstos en el momento en que se han incorporado a los institutos catalanes (Serra \& Palaudàrias, 2010).

Este proyecto surgió a partir de observar el aumento del volumen de población inmigrante y su distribución en el territorio catalán durante los últimos años. A partir de diferentes estudios, algunos de ellos ya nombrados, se pudo constatar que de toda esta población de origen extranjero solo un por-

\section{Introduction}

Since the 90s, the flow arrival of immigrants in Spain led to a rapid increase in the participation of immigrant students in the education system. In recent years, before the economic crisis, the presence of foreign students in compulsory education increased over $500 \%$. This phenomenon has been particularly noticeable in Catalonia, where during 2008-2009 15.9\% of students in schools were foreigners, compared to $11.6 \%$ foreigner's students in all Spain, according to the Ministry of Education (2010).

Unfortunately, in some cases, these migration processes experienced by these immigrant students have a negative impact on their academic trajectories either by the ruptures that occurred as a result of these changes, by the bereavement situation some of these children and youth have to live, or by the difficulties they face at the time they join the Catalan schools (Serra \& Palaudàries, 2010).

Therefore, this project emerged from observing the increased number of immigrant population and its distribution in Catalonia in recent years. From various studies, some of them already mentioned, it was found that from all this foreign population only a very small percentage reached college. For example the Report oq from the Catalan institute of Infancia i Món Urbà (2009) showed that $44.3 \%$ of immigrants have failed to post-compulsory education (in front of a $31 \%$ average for the youth of our country). Thus, the aim 
centaje muy reducido llegaba a la universidad. Por ejemplo en el Informe 09 del Instituto de Infancia i Món Urbà (2009), observamos que un $44,3 \%$ de los inmigrantes no había conseguido la educación postobligatoria (delante de un 31\% de media de los jóvenes de nuestro país). De esta manera se centró el objetivo de esta investigación en conocer las competencias y los factores más relevantes que los estudiantes universitarios hijos/as de familias inmigrantes consideran que les han sido útiles para conseguir este éxito escolar, entendiendo éxito como el hecho de llegar a la universidad.

\section{Justificación y objetivos}

Miles de estudiantes en Cataluña y España se enfrentan con el problema del fracaso escolar, que afecta a un tercio de los jóvenes que se encuentran en edad de completar los estudios obligatorios (Estellés et al., 2012). Según el informe PISA 2009, España esta significativamente por debajo de la media de los países de la OCDE en cuanto al nivel de aprendizaje de los estudiantes. Concretamente, en Cataluña, el $29 \%$ de los jóvenes entre 18 y 24 años no finaliza sus estudios post obligatorios, lejos de la media de un 14\% de la UE-27 y todavía más del objetivo de la Estrategia Europa 2020 (que pretende que sea del 10\%) (Truño, 2012). En el caso de la población inmigrada, las cifras suben hasta el $45 \%$ (Estellés et al., 2012).

En el caso concreto de la educación secundaria no obligatoria, se aprecia que la representación de jóvenes de nacionalidad extranjera dentro de ésta es considerablemente más baja que la de los otros estudiantes (Alarcón, 2007). Muchos estudiantes de secundaria de origen inmigrante tienen unas claras expectativas de continuación de los estudios dentro de los ciclos formativos o de inserción laboral una vez finalizados los estudios obligatorios. De esta manera, los hijos e hijas de las personas extranjeras se incorporan antes dentro del mercado laboral y en mayor medida que los jóvenes nativos, tal y como aseguraba el Informe de Juventud en España del 2004, ya que tienen tendencia a seguir las pautas propias de incorporación al mundo laboral de la clase obrera o de los estratos económicos más bajos, especialmente los jóvenes dominicanos y los marroquíes (Aparicio \& Tornos, 2006). El abandono of this research is focused on understanding the most important competencies and factors that college students from immigrant families feel have been useful for their educational success, educational success seen as the enrolment in programs beyond compulsory education.

\section{Justification and goals}

Thousands of students in Catalonia and Spain are faced with the problem of school failure, which affects a third of young people in the age of completing compulsory education (Estellés et al., 2012). According to the PISA 2009 report, Spain is significantly below the average of the OECD countries in the level of students' learning. Specifically, in Catalonia, 29\% of young people aged 18 to 24 years old do not complete their post-compulsory studies, far from the average of $14 \%$ of the EU-27 and much further from the goal of the Europe 2020 strategy (which aims to be 10\%) (Truño, 2012). In the case of the immigrant population, the figures rise to $45 \%$ (Estellés et al., 2012).

In the case of upper higher education, is seen how young people of foreign nationality are considerably lower represented within this level than the other students (Alarcón, 2007). Many high school students from immigrant backgrounds have clear expectations of continuing education within training cycles or expectations of job placement after completing compulsory education. Thus, children of foreigners are incorporated into the labour market earlier and to a greater extent than Spanish youths, as the Youth Report in Spain claimed (2004). This group of people tend to follow the guidelines for the incorporation into the labour market of the working class or of the lower economic strata, especially young Dominicans, followed by Moroccans (Aparicio \& Tornos, 2006). Early school leavers (or ESL), a low level of education or the lack of specific training has an impact on the personal and professional future of

[ 266 ] ELENA CANO GARCÍA Y MAITE FERNÁNDEZ FERRER 
escolar prematuro, un nivel de estudios bajo o la falta de formación profesional específica tiene un impacto claro sobre el futuro personal y profesional de los jóvenes (Navarrete, 2007).

El estudio CIDE-MEC (2005), por otra parte, reconoce que la escuela actual no está hecha todavía desde un modelo intercultural sino desde un modelo culturalmente homogeneizador, por lo que la diversidad cultural está vista como un problema o déficit. A pesar del acceso universal y del derecho a la educación, no se garantiza de la misma manera la calidad y el aprovechamiento para todos los grupos de estudiantes, con lo que pueden aumentar las diferencias ya existentes. En relación con esto, algunos estudios (Aja et al., 2000) analizan la distribución de la población inmigrante en centros, alertando de la inequidad que puede existir debajo de los lemas que defienden la elección del centro y enfatizan la importancia de una buena política distributiva de la población recién llegada.

En conclusión, la reducción del fracaso escolar del colectivo inmigrante es decisiva a la hora de reproducir el ciclo vicioso de pobreza y exclusión social en la etapa adulta. Por este motivo, en la garantía del derecho a la educación las sociedades se juegan mucho ya que la vulneración al mismo podría ofrecer un escenario preocupante de cara a los próximos años desde el punto de vista de la distribución de las oportunidades sociales y educativas. En la mayoría de nuestras sociedades se espera que sea el sistema educativo el que iguale estas diferencias.

Así, este proyecto partía de un único objetivo inicial: conocer las competencias clave (con relación a las 8 competencias establecidas por la LOE) y los factores más relevantes (con relación a las variables identificadas por la literatura sobre éxito escolar) que los estudiantes universitarios hijos/as de familias inmigrantes consideran que les han sido primordiales a la hora de conseguir su éxito educativo, entendiendo este como el hecho de llegar a la universidad.

En consecuencia, este proyecto integra campos de estudio diferentes: las competencias clave, la inclusión de la población inmigrante, los factores que influyen en el éxito escolar. El conjunto de los estudios sobre estos tres ejes (OCDE, 1998; Generalitat de Catalunya, 2005; Murillo, 2005; Alegre, Benito y González, 2006; Monereo, 2009; Instituto de In- young people. An impact that mainly results in a shortfall of resources to overcome the pursuit of a quality job, or in a precariousness to adapt to an increasingly demanding and unstable labour market (Navarrete, 2007).

The CIDE-MEC study (2005) for example, recognizes that the current school is not yet made within an intercultural model but from a culturally homogenizing model, which sees cultural diversity as a problem or deficit. Despite the universal right of access to education, this is not guaranteed in the same way of quality and achievement for all groups of students. This may increase the existing differences.

Relating to that, some studies (Aja et al., 2000) analysed the distribution of the immigrant population in schools, warning of the inequality that may exist under the slogans advocating the families' own election of the education centre. This emphasizes the importance of a good policy to distributive the newcomer population in the education system.

In conclusion, the reduction of school failure of the immigrants is decisive when playing the vicious cycle of poverty and social exclusion in adulthood. For this reason, in ensuring the right to education societies much take into account that with the violation of this right a worrying scenario could be offered over the coming years from the point of view of the distribution of social and educational opportunities. In most of our societies is expected to be the education system that match these differences. The fact that children of immigrant families do not amount to exceed compulsory education follows that those same people have not acquired the basic competencies required by the labour market and therefore they will find it very difficult to achieve social inclusion.

Thus, this project started from a single initial goal: to identify the key factors and competencies that college students from immigrant families feel have been useful for their educational success, educational success seen as the enrolment in programs beyond compulsory education.

Consequently, this project integrates different fields of study: the key competencies, the inclusion of the immigrant population and the factors influencing academic success, 
fancia i Món Urbà, 2009; Comisión Europea, 2010; Serra y Palaudàrias, 2010; Oseguera, Conchas \& Mosqueda, 2011) indican qué variables están más vinculadas al éxito escolar y por este motivo, desde el proyecto que se presenta a continuación, se ha querido descubrir si estos elementos emergían de los relatos y narraciones de vida de los jóvenes participantes del estudio y cómo los exponían los protagonistas.

\section{Metodología}

El estudio, de naturaleza interpretativa, pretende la comprensión profunda de los elementos que, a juicio de cada individuo, le ayudaron a desarrollar sus competencias básicas y de los factores que pudieron contribuir a conseguir el éxito escolar en el contexto catalán. Por este motivo, la metodología fue eminentemente cualitativa y el enfoque básicamente fenomenológico, empleándose tres fuentes diferentes de recogida de información: los relatos de vida, las narraciones audiovisuales y el cuestionario. Concretamente, para este artículo, nos referimos a los resultados analizados a través de los relatos de vida.

Para la selección de los participantes se buscaron personas que estuvieran realizando sus estudios universitarios y que hubieran hecho toda o parte de la escolaridad básica en nuestro país, aspecto que llevó a desestimar muchos informantes. Por este motivo, a causa de esta especificidad en los criterios de selección, se hace necesario destacar la dificultad para encontrar estudiantes con el perfil solicitado y que además quisieran formar parte del estudio. Es necesario, pues, destacar la dedicación para la búsqueda de informantes, así como las acciones que se desarrollaron para su identificación y la petición de colaboración, entre las que destacaron: carta a la Oficina de Preinscripciones para las Pruebas de Acceso a la Universidad; carta a la vicerrectora de estudiantes de la Universitat de Barcelona; llamada a la participación a través de las asociaciones de inmigrantes; llamada a través del campus virtual de la Universitat de Barcelona; carteles informativos sobre la investigación pidiendo colaboraciones colgados en distintas facultades de la Universitat de Barcelona; llamada a la participación informal a partir de contactos de los miembros
(OCDE, 1998; Generalitat de Catalunya, 2005; Murillo, 2005; Alegre, Benito y González, 2006; Monereo, 2009; Instituto de Infancia i Món Urbà, 2009; Comisión Europea, 2010; Serra y Palaudàrias, 2010; Oseguera, Conchas \& Mosqueda, 2011) studying these elements by life stories and the development of audio-visual narratives.

\section{Methodology}

This study of interpretive nature seeks a deep understanding of the elements that, according to each individual, helped to develop their basic competencies and the key factors that might contribute to achieving academic success. For this reason, the methodology was essentially qualitative and the approach was basically phenomenological, using three different sources of data collection: life stories, audio-visual narratives and questionnaire. Specifically, for this article we refer to the results analysed through life stories.

For the selection of participants people who were in higher education were sought with the need of them having done all or part of the basic education in our country, something that led to dismiss many informants. Therefore, because of this specificity in the selection criteria, it is necessary to highlight the difficulty in finding students with the required profile and who also would wanted to be part of the study. Finally, participants were selected based on the following specific criteria:

1. Age of arrival: students who performed some grade of the compulsory education level in our country. Therefore, these were students who arrived before the age of sixteen.

2. Nationality: students from Latin America, Africa or Asia or non-EU Europe.

3. Variety of universities and degrees.

Obtaining information was conducted in 2010 by the contributions of student's children of immigrants. In order to know what key competencies and the most relevant factors those students from immigrant families feel they have been help-

[ 268 ] ELENA CANO GARCÍA Y MAITE FERNÁNDEZ FERRER 
del equipo; información presencial en las aulas de la Facultad de Pedagogía y de Formación del Profesorado; correos electrónicos a personas clave por su perfil profesional y lugar de trabajo; carta a la Secretaria de Inmigración de la Generalitat de Cataluña; contacto con el Departamento de Acción Social y Ciudadanía de la Generalitat de Cataluña; contacto con el programa de televisión "De aquí, de allá" de la Televisión de Cataluña. Finalmente, tras un complejo proceso de búsqueda, los participantes estuvieron seleccionados en función de los siguientes criterios específicos:

1. Edad de llegada: los estudiantes que realizaron algún curso de educación obligatoria en el país de llegada. Por lo tanto, eran estudiantes que llegaron antes de los dieciséis años.

2. Nacionalidad: los estudiantes provenían de América Latina, África o Asia, y/o Europa no comunitaria.

3. Variedad de universidades y titulaciones.

La obtención de la información se llevó a cabo durante el año 2010 mediante las aportaciones de los estudiantes hijos/as de inmigrantes de Cataluña. Para poder llevar a cabo el objetivo de conocer cuáles son las competencias clave y los factores más relevantes que los estudiantes universitarios hijos/as de familias inmigradas consideran que les han sido útiles para conseguir el éxito educativo el estudio se centró en las preguntas que en todo momento orientaron las contribuciones de los informantes:

- ¿Cuáles crees que han sido los principales factores o variables que te han ayudado a llegar a la universidad?

- ¿Cuáles son los principales obstáculos con los que te has encontrado? ¿Cómo los has podido superar?

Solo se les informó sobre los tres ejes de la investigación (las competencias clave, el éxito escolar y la inclusión educativa) que orientarían nuestro análisis, dejando totalmente libre las aportaciones de los productos pertinentes (tanto en el caso de los relatos de vida como en las narraciones audiovisuales). Las condiciones que se pidieron a los participantes fueron que la extensión del escrito podía ser absolutamente libre y solo se fijó una fecha para la entrega del documento. ful for their academic success the study focused on the following questions:

- What do you think have been the main factors or variables that have helped you get to college?

- What are the main obstacles you have encountered? How could you overcome them?

Informants were only informed about the three axes of research (key competencies, school success and educational inclusion) that would guide our analysis, leaving completely free the contributions of relevant products (both in case of life stories and audio-visual narratives).

The conditions that participants were asked for were that the extension of writing could be absolutely free and only a date for the delivery of the life stories was fixed.

Later these two data collection strategies within this qualitative methodology were complemented with the development and administration of a survey. 
Estas dos estrategias de recogida de información dentro de esta metodología cualitativa estuvieron complementadas con la elaboración y administración de un cuestionario.

Finalmente se obtuvieron 16 relatos de vida de los que 3 fueron desestimados por no cumplir

Tabla 1. Instrumentos de recogida de información

\begin{tabular}{|l|c|}
\hline Instrumentos & Valor \\
\hline Relatos de vida & 13 \\
\hline Narraciones audiovisuales & 4 \\
\hline Cuestionarios & 128 \\
\hline
\end{tabular}

Table 1. Data collection instruments

\begin{tabular}{|l|c|}
\hline Instruments & Number \\
\hline Life stories & 13 \\
\hline Audio-visual narratives & 4 \\
\hline Survey & 128 \\
\hline
\end{tabular}

Tabla 2. Listado de informantes de los relatos de vida

\begin{tabular}{|l|c|c|c|l|}
\hline $\begin{array}{l}\text { Nombre y } \\
\text { apellidos }\end{array}$ & $\begin{array}{c}\text { País de } \\
\text { procedencia }\end{array}$ & $\begin{array}{c}\text { Nacionalidad } \\
\text { paterna }\end{array}$ & $\begin{array}{c}\text { Nacionalidad } \\
\text { materna }\end{array}$ & Estudios \\
\hline A.A.K. & Marruecos & Marruecos & Marruecos & Enfermería \\
\hline S.S.K. & Brasil & Brasil & Brasil & Pedagogía \\
\hline O.O. & España & Nigeria & Filipinas & Administración y Dirección de Empresas \\
\hline D.L.H. & Marruecos & Marruecos & Marruecos & Educación Infantil \\
\hline H.L. & Marruecos & Marruecos & Marruecos & Educación Social \\
\hline K.Y.M. & España & Marruecos & Marruecos & Psicología \\
\hline A.B.M. & Marruecos & Marruecos & Marruecos & Máster en Intervenciones Sociales y Educativas \\
\hline N.V.T. & España & Vietnam & Vietnam & Criminología \\
\hline H.S. & Marruecos & Marruecos & Marruecos & Estudios Ingleses \\
\hline G.D.N. & España & Méjico & España & Posgrado en Educación Emocional \\
\hline V.A.A. & Romania & Romania & Romania & Sociología \\
\hline A.E. & Uruguay & Uruguay & Uruguay & Ciencias Políticas \\
\hline P.W.A. & Argentina & Cataluña & Argentina & Psicología \\
\hline
\end{tabular}

Table 2. List of informants of the life stories

\begin{tabular}{|l|c|c|c|l|}
\hline $\begin{array}{l}\text { Name and } \\
\text { surname }\end{array}$ & $\begin{array}{c}\text { Home } \\
\text { country }\end{array}$ & $\begin{array}{c}\text { Father natio- } \\
\text { nality }\end{array}$ & $\begin{array}{c}\text { Mother natio- } \\
\text { nality }\end{array}$ & Degree \\
\hline A.A.K. & Morocco & Morocco & Morocco & Nursery \\
\hline S.S.K. & Brazil & Brazil & Brazil & Pedagogy \\
\hline O.O. & Spain & Nigeria & Filipinas & Administration and Business Management \\
\hline D.L.H. & Morocco & Morocco & Morocco & Primary Education \\
\hline H.L. & Morocco & Morocco & Morocco & Social Services \\
\hline K.Y.M. & Spain & Morocco & Morocco & Psychology \\
\hline A.B.M. & Morocco & Morocco & Morocco & Master in Social and Educational Action \\
\hline N.V.T. & Spain & Vietnam & Vietnam & Criminology \\
\hline H.S. & Morocco & Morocco & Morocco & English Studies \\
\hline G.D.N. & Spain & Mexico & Spain & Master in Emotional Education \\
\hline V.A.A. & Romania & Romania & Romania & Sociology \\
\hline A.E. & Uruguay & Uruguay & Uruguay & Political Sciences \\
\hline P.W.A. & Argentina & Catalonia & Argentina & Psychology \\
\hline
\end{tabular}

[ 270 ] ELENA CANO GARCÍA Y MAITE FERNÁNDEZ FERRER 
con los dos criterios fundamentales referidos a la edad de llegada y a la nacionalidad, y por lo tanto, se han analizado 13 relatos escritos. Los documentos tenían una media de tres páginas (uno de ellos incluía fotografías de su familia y de su país de origen).

Se garantizó la confidencialidad en el trato de los datos recogidos en los relatos de vida. El análisis documental se realizó siguiendo un proceso inductivo-deductivo. A partir de la lectura de todos los relatos de vida por parte de seis miembros del grupo de investigación se identificaron las unidades de significado consideradas más relevantes para finalmente llegar a un listado único de unidades agrupadas en 10 categorías, con las que analizar y clasificar el contenido de todos los relatos que se presentan en el apartado de resultados de este artículo. Esta categorización, aparte de que permitía cuantificar la aparición de las unidades de significado, como se explicará más adelante, permitía organizar categoría a categoría y unidad a unidad el conjunto de fragmentos que hemos considerado que hacen referencia a eje de estudio.

Para el análisis de los relatos de vida se utilizó el programa Atlas.ti a través del cual se obtuvo la tabla de frecuencias (número de veces que sale la unidad en el conjunto de los 13 relatos) así como el análisis numérico (el total de veces que han salido las unidades emergentes en todos los relatos). En la tabla que se presenta a continuación se puede observar este análisis y sombreado en gris aparecen las unidades más frecuentes y que, además, aparecen en más relatos.
Finally 16 life stories were obtained of which 3 were dismissed for failing to meet the two fundamental criteria for the selection of the participants relating to their age of arrival and nationality. Therefore, 13 life stories have been analysed. These documents had an average of three pages each (one of them included photographs of his family and his home country).

The documentary analysis was performed following an inductive process. From reading all the life stories by six members of the research group meaning units considered most relevant were identified. From this finally a single list of items grouped into 10 categories was created with which to analyse and classify the content of all the life stories presented in the results section of this article.

For the analysis of life stories the Atlas.ti program was used whereby two documents and evidences of the most important results were obtained. On the one hand the frequency table with the information on how often every item ran (belonging to a category of the ten existing) in each life story. Thus, as it can be seen below, in the left of the table there are all the items or meaning units, as well as how many times each one has appeared in every life stories and the total times it has appeared in all the narratives in total. 
Tabla 3. Evidencia de los resultados de los relatos de vida a través del programa Atlas.ti

\begin{tabular}{|c|c|c|c|}
\hline & $\begin{array}{l}\text { Frecuencia } \\
\text { total }\end{array}$ & \multicolumn{2}{|c|}{$\begin{array}{c}\text { Número (y \%) de relatos } \\
\text { en las que aparece }\end{array}$} \\
\hline \multicolumn{4}{|c|}{ 1. LAS COMPETENCIAS COMO EJES CLAVE PARA SU PROGRESIÓN } \\
\hline 1.1. Capacidad de comunicarse & 11 & $6 / 13$ & $46,15 \%$ \\
\hline 1.2. Comunicación en lengua extranjera & 6 & $4 / 13$ & $30,77 \%$ \\
\hline 1.3. Competencia matemática y en ciencia y tecnología & 5 & $3 / 13$ & $23,08 \%$ \\
\hline 1.4. Competencia digital & O & O & ○\% \\
\hline 1.5. Aprender a aprender & 5 & $3 / 13$ & $23,08 \%$ \\
\hline 1.6. Competencias sociales y cívicas & 13 & $8 / 13$ & $61,54 \%$ \\
\hline 1.7. Sentido de la iniciativa y autonomía & 10 & $6 / 13$ & $46,15 \%$ \\
\hline 1.8. Conciencia y expresión cultural & 4 & $3 / 13$ & $23,8 \%$ \\
\hline \multicolumn{4}{|l|}{ 1.9. Competencias emocionales } \\
\hline 1.9.1. Auto exigencia intrínseca & 6 & $6 / 13$ & $46,15 \%$ \\
\hline 1.9.2. Alta auto exigencia debido al esfuerzo de las familias & 9 & $5 / 13$ & $38,46 \%$ \\
\hline 1.9.3. Auto eficacia: sentirse capaz y tener seguridad en el mismo & 7 & $5 / 13$ & $38,46 \%$ \\
\hline 1.9.4. Cultura del esfuerzo y responsabilidad & 18 & $9 / 13$ & $69,23 \%$ \\
\hline \multicolumn{4}{|c|}{ 2. ELEMENTOS FAVORECEDORES Y ACCIONES QUE PUEDEN INFLUIR EN EL ÉXITO } \\
\hline \multicolumn{4}{|c|}{ 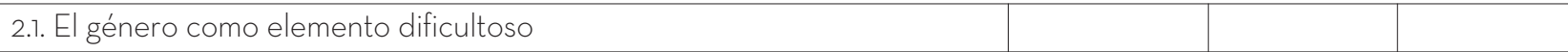 } \\
\hline 2.1.7. Presión por el género & 10 & $6 / 13$ & $46,15 \%$ \\
\hline \multicolumn{4}{|l|}{ 2.2. Sentimientos relacionados con el éxito } \\
\hline 2.2.1. Hecho de sentirse arraigado & 1 & $1 / 13$ & $7,69 \%$ \\
\hline 2.2.2. Auto percepción positiva & 11 & $8 / 13$ & $61,54 \%$ \\
\hline 2.2.3. Auto percepción de no ser diferente & 8 & $6 / 13$ & $46,15 \%$ \\
\hline 2.2.4. Ser el primer universitario de la familia & 5 & $5 / 13$ & $38,46 \%$ \\
\hline 2.2.5. Expectativas de futuro: ir a la universidad & 14 & $8 / 13$ & $61,54 \%$ \\
\hline 2.2.6. Capacidad de alejarse de las "malas amistades" & 3 & $3 / 13$ & $23,08 \%$ \\
\hline 2.2.7. Ganes de aprender: educación como oportunidad & 11 & $6 / 13$ & $46,15 \%$ \\
\hline 2.2.8. "Ganarse el respeto" de los otros a través del éxito escolar & 8 & $6 / 13$ & $46,15 \%$ \\
\hline 2.2.9. Valoración de la propia cultura & 1 & $1 / 13$ & $7,69 \%$ \\
\hline \multicolumn{4}{|l|}{ 2.3. Diferencia de percepciones en función de los niveles educativos } \\
\hline 2.3.1. Las percepciones de la escolaridad en la E. Infantil & 4 & $3 / 13$ & $23, \circ 8 \%$ \\
\hline \multicolumn{4}{|l|}{ 2.3.2. Las percepciones de la escolaridad en la Educación Primaria: } \\
\hline 2.3.2.1. Recuerdos de inclusión infantil & 6 & $6 / 13$ & $46,15 \%$ \\
\hline 2.3.2.2. Recuerdos de exclusión infantil & 1 & $1 / 13$ & $7,69 \%$ \\
\hline 2.3.3. Las percepciones de la escolaridad en la E. Secundaria & 10 & $9 / 13$ & $69,23 \%$ \\
\hline \multicolumn{4}{|l|}{ 2.4. La experiencia universitaria } \\
\hline 2.4.1. Las percepciones de la escolaridad en la universidad & 1 & $1 / 13$ & $7,69 \%$ \\
\hline 2.4.2. El desconocimiento del entorno universitario & 3 & $2 / 13$ & $15,39 \%$ \\
\hline 2.4.3. La diferencia entre acceder y mantenerse & 2 & $1 / 13$ & $7,69 \%$ \\
\hline 2.4.4. Haber de compaginarla, por necesidad, con un trabajo & 5 & $3 / 13$ & $23,08 \%$ \\
\hline 2.4.5. Los objetivos de llegar a la universidad & 5 & $5 / 13$ & $38,46 \%$ \\
\hline
\end{tabular}


Table 3. Evidence of the results of the life stories through Atlas.ti

\begin{tabular}{|c|c|c|c|}
\hline & Frequency & \multicolumn{2}{|c|}{ Numerical analysis } \\
\hline \multicolumn{4}{|l|}{ 1. COMPETENCIES AS KEY FACTORS FOR ACADEMIC SUCCESS } \\
\hline 1.1. Ability to communicate & 11 & $6 / 13$ & $46,15 \%$ \\
\hline 1.2. Communication in foreign languages & 6 & $4 / 13$ & $30,77 \%$ \\
\hline 1.3. Mathematics, science and technology knowledge & 5 & $3 / 13$ & $23,08 \%$ \\
\hline 1.4. Digital competency & O & O & ०\% \\
\hline 1.5. Learning to learn & 5 & $3 / 13$ & $23,08 \%$ \\
\hline 1.6. Social and civic competencies & 13 & $8 / 13$ & $61,54 \%$ \\
\hline 1.7. Sense of initiative and autonomy & 10 & $6 / 13$ & $46,15 \%$ \\
\hline 1.8. Cultural awareness and expression & 4 & $3 / 13$ & $23,8 \%$ \\
\hline \multicolumn{4}{|l|}{ 1.9. Emotional competencies } \\
\hline 1.9.1. Auto intrinsic requirement & 6 & $6 / 13$ & $46,15 \%$ \\
\hline 1.9.2. High auto demand due to the efforts of families & 9 & $5 / 13$ & $38,46 \%$ \\
\hline 1.9.3. Self-efficacy: feel capable and have the same security & 7 & $5 / 13$ & $38,46 \%$ \\
\hline 1.9.4. Dedication and personal effort & 18 & $9 / 13$ & $69,23 \%$ \\
\hline \multicolumn{4}{|c|}{ 2. ELEMENTS AND ACTIONS THAT CAN INFLUENCE ON SCHOOL SUCCESS } \\
\hline \multicolumn{4}{|c|}{\begin{tabular}{|l|l} 
2.1. Gender as a difficult element & \\
\end{tabular}} \\
\hline 2.1.7. Pressure by gender & 10 & $6 / 13$ & $46,15 \%$ \\
\hline \multicolumn{4}{|l|}{ 2.2. Feelings related to success } \\
\hline 2.2.1. To feel part of & 1 & $1 / 13$ & $7,69 \%$ \\
\hline 2.2.2. Auto positive perception & 11 & $8 / 13$ & $61,54 \%$ \\
\hline 2.2.3. Self perception of not being different & 8 & $6 / 13$ & $46,15 \%$ \\
\hline 2.2.4. Being the first college person in the family & 5 & $5 / 13$ & $38,46 \%$ \\
\hline 2.2.5. Expectations for the future: going to college & 14 & $8 / 13$ & $61,54 \%$ \\
\hline 2.2.6. Ability to get away from the certain "friends" & 3 & $3 / 13$ & $23,08 \%$ \\
\hline 2.2.7. Eager to learn: education as opportunity & 11 & $6 / 13$ & $46,15 \%$ \\
\hline 2.2.8. "Earning the respect" of others through school success & 8 & $6 / 13$ & $46,15 \%$ \\
\hline 2.2.9. Valuing the own culture & 1 & $1 / 13$ & $7,69 \%$ \\
\hline \multicolumn{4}{|c|}{ 2.3. Differences between perceptions depending on the educational levels } \\
\hline 2.3.1. Perceptions of schooling in elementary education & 4 & $3 / 13$ & $23,08 \%$ \\
\hline \multicolumn{4}{|l|}{ 2.3.2. Perceptions of schooling in primary education } \\
\hline 2.3.2.1. Memories of childhood inclusion & 6 & $6 / 13$ & $46,15 \%$ \\
\hline 2.3.2.2. Memories of childhood exclusion & 1 & $1 / 13$ & $7,69 \%$ \\
\hline 2.3.3. Perceptions of schooling in high school & 10 & $9 / 13$ & $69,23 \%$ \\
\hline \multicolumn{4}{|l|}{ 2.4. The college experience } \\
\hline 2.4.1. Perceptions of schooling in college & 1 & $1 / 13$ & $7,69 \%$ \\
\hline 2.4.2. Not knowing the university environment & 3 & $2 / 13$ & $15,39 \%$ \\
\hline 2.4.3. The difference between access and remain & 2 & $1 / 13$ & $7,69 \%$ \\
\hline 2.4.4. Having to combine it with a job & 5 & $3 / 13$ & $23,08 \%$ \\
\hline 2.4.5. The goals of going to college & 5 & $5 / 13$ & $38,46 \%$ \\
\hline
\end{tabular}




\begin{tabular}{|c|c|c|c|}
\hline & $\begin{array}{l}\text { Frecuencia } \\
\text { total }\end{array}$ & \multicolumn{2}{|c|}{$\begin{array}{c}\text { Número (y \%) de relatos } \\
\text { en las que aparece }\end{array}$} \\
\hline \multicolumn{4}{|l|}{ 3. LOS ELEMENTOS FAVORECEDORES DE LA INCLUSIÓN SOCIAL } \\
\hline \multicolumn{4}{|l|}{ 3.1. Elementos favorecedores relativos a la familia } \\
\hline 3.1.1. Expectativas de la familia & 15 & $8 / 13$ & $61,54 \%$ \\
\hline 3.1.2. Acciones para dar soporte en la educación de sus hijos/as & 71 & $6 / 13$ & $46,15 \%$ \\
\hline 3.1.3. Valoración positiva que otorga la familia a la educación & 16 & $9 / 13$ & $69,23 \%$ \\
\hline 3.1.4. Los estudios previos de los padres & 6 & $4 / 13$ & $30,77 \%$ \\
\hline 3.1.5. Una figura familiar crucial: la madre & 7 & $4 / 13$ & $30,77 \%$ \\
\hline 3.1.6. Una figura familiar crucial: los hermanos & 9 & $5 / 13$ & $38,46 \%$ \\
\hline 3.1.7. Una figura "familiar" crucial: la pareja & 1 & $1 / 13$ & $7,69 \%$ \\
\hline \multicolumn{4}{|l|}{ 3.2. Elementos favorecedores relativos al proceso de inmigración } \\
\hline 3.2.1. Expectativas antes de la inmigración & 2 & $2 / 13$ & $15,39 \%$ \\
\hline 3.2.2. Consecuencias y efectos positivos de tener familia en el país & 1 & $1 / 13$ & $7,69 \%$ \\
\hline 3.2.3. Ser los primeros inmigrantes del pueblo/ ciudad/ barrio & 3 & $2 / 13$ & $15,39 \%$ \\
\hline 3.2.4. Entender el vivir con otras culturas como enriquecedor & 4 & $4 / 13$ & $30,77 \%$ \\
\hline 3.2.5. Mantenimiento de la identidad cultural & 4 & $3 / 13$ & $23,08 \%$ \\
\hline \multicolumn{4}{|l|}{ 4. LOS ELEMENTOS DIFICULTADORES DE LA INCLUSIÓN SOCIAL } \\
\hline \multicolumn{4}{|l|}{ 4.1. Elementos dificultadores relativos a la familia } \\
\hline 4.1.1. Las pocas expectativas de la familia & 6 & $1 / 13$ & $7,69 \%$ \\
\hline 4.1.2. Los recursos económicos limitados & 11 & $7 / 13$ & $53,85 \%$ \\
\hline 4.1.3. La religión familiar & 10 & $4 / 13$ & $30,77 \%$ \\
\hline 4.1.4. El papel del estudiante dentro de la estructura familiar & 2 & $2 / 13$ & $15,39 \%$ \\
\hline \multicolumn{4}{|l|}{ 4.2. Elementos dificultadores relativos al proceso de inmigración } \\
\hline 4.2.1. Primer choque cultural: diferencias en los hábitos & 5 & $3 / 13$ & $23,08 \%$ \\
\hline 4.2.2. Percepción de las diferencias y situaciones de discriminación & 12 & $7 / 13$ & $53,85 \%$ \\
\hline \multicolumn{4}{|l|}{ 4.3. Elementos dificultadores relativos a la vivienda } \\
\hline 4.3.1. Dificultades derivadas de la situación doméstica & 2 & $2 / 13$ & $15,39 \%$ \\
\hline 4.3.2. Situación de la vivienda familiar y el tener que desplazarse & 5 & $4 / 13$ & $30,77 \%$ \\
\hline 4.3.3. Diferencia entre entorno urbano y rural & 3 & $3 / 13$ & $23,08 \%$ \\
\hline \multicolumn{4}{|c|}{ 5. LA ESCUELA COMO ELEMENTO CRUCIAL PARA EL ÉXITO ESCOLAR } \\
\hline \multicolumn{4}{|c|}{ 5.1. El impacto positivo de un buen proceso de adaptación } \\
\hline 5.1.1. Importancia del conocimiento previo de algunas personas & 1 & $1 / 13$ & $7,69 \%$ \\
\hline 5.1.2. Existencia de otros estudiantes inmigrantes en el centro & 4 & $4 / 13$ & $30,77 \%$ \\
\hline 5.1.3. Superar el cambio de etapa educativa y de centro & 6 & $4 / 13$ & $30,77 \%$ \\
\hline 5.1.4. Superar el desconocimiento de los espacios y las personas & 14 & $6 / 13$ & $46,15 \%$ \\
\hline \multicolumn{4}{|l|}{ 5.2. Los sujetos del centro escolar: el profesorado } \\
\hline 5.2.1. Las altas expectativas del profesorado & 15 & $7 / 13$ & $53,85 \%$ \\
\hline 5.2.2. Las características del buen profesor & 34 & $17 / 13$ & $84,62 \%$ \\
\hline \multicolumn{4}{|l|}{ 5.2.3. Acciones de profesorado: } \\
\hline 5.2.3.1. El refuerzo positivo por parte del profesorado & 5 & $3 / 13$ & $23,08 \%$ \\
\hline 5.2.3.2. La intervención del profesorado más allá de lo académico & 6 & $5 / 13$ & $38,46 \%$ \\
\hline 5.2.3.3. El interés por conocer la cultura del inmigrante & 1 & $1 / 13$ & $7,69 \%$ \\
\hline 5.3. Los sujetos del centro escolar: el equipo directivo & 5 & $4 / 13$ & $30,77 \%$ \\
\hline \multicolumn{4}{|l|}{ 5.4. Soluciones organizativas de la escuela } \\
\hline 5.4.1. El aula de acogida & 15 & $3 / 13$ & $23,08 \%$ \\
\hline 5.4.2. Las metodologías inclusivas & 2 & $1 / 13$ & $7,69 \%$ \\
\hline 5.4.3. Las clases de repaso & 4 & $2 / 13$ & $15,39 \%$ \\
\hline 5.4.4. Los agrupamientos heterogéneos & 4 & $4 / 13$ & $30,77 \%$ \\
\hline
\end{tabular}

[ 274 ] ELENA CANO GARCÍA Y MAITE FERNÁNDEZ FERRER

SIPS - PEDAGOGIA SOCIAL. REVISTA INTERUNIVERSITARIA [1139-1723 (2015) 26, 263-284] TERCERA ÉPOCA 


\begin{tabular}{|c|c|c|c|}
\hline & Frequency & \multicolumn{2}{|c|}{ Numerical analysis } \\
\hline \multicolumn{4}{|l|}{ 3. KEY FACTORS FOR SOCIAL INCLUSION } \\
\hline \multicolumn{4}{|l|}{ 3.1. Supporting elements relating to the family } \\
\hline 3.1.1. Family expectations & 15 & $8 / 13$ & $61,54 \%$ \\
\hline 3.1.2. The actions of the family to support the education of their children & 11 & $6 / 13$ & $46,15 \%$ \\
\hline 3.1.3. The positive value that gives the family on the importance of education & 16 & 9/13 & $69,23 \%$ \\
\hline 3.1.4. The previous family qualifications & 6 & $4 / 13$ & $30,77 \%$ \\
\hline 3.1.5. A familiar capital figure: the relationship with the mother & 7 & $4 / 13$ & $30,77 \%$ \\
\hline 3.1.6. A familiar capital figure: the relationship with the siblings & 9 & $5 / 13$ & $38,46 \%$ \\
\hline 3.1.7. A familiar capital figure: the relationship with the boyfriend or girlfriend & 1 & $1 / 13$ & $7,69 \%$ \\
\hline \multicolumn{4}{|l|}{ 3.2. Predisposing factors relating to the migration process } \\
\hline 3.2.1. Expectations before the migration process & 2 & $2 / 13$ & $15,39 \%$ \\
\hline 3.2.2. Consequences and positive effects of having family in the country & 1 & $1 / 13$ & $7,69 \%$ \\
\hline 3.2.3. Being the first immigrants of the town, city or district & 3 & $2 / 13$ & $15,39 \%$ \\
\hline 3.2.4. Understanding living with other cultures as enriching & 4 & $4 / 13$ & $30,77 \%$ \\
\hline 3.2.5. Maintenance of cultural identity & 4 & $3 / 13$ & $23,08 \%$ \\
\hline \multicolumn{4}{|l|}{ 4. HAMPERING FACTORS FOR SOCIAL INCLUSION } \\
\hline \multicolumn{4}{|l|}{ 4.1. Hampering factors relating to the family } \\
\hline 4.1.1. The lack of family expectations & 6 & $1 / 13$ & $7,69 \%$ \\
\hline 4.1.2. Limited financial resources & 11 & $7 / 13$ & $53,85 \%$ \\
\hline 4.1.3. The family religion & 10 & $4 / 13$ & $30,77 \%$ \\
\hline 4.1.4. The role of the children within the family structure & 2 & $2 / 13$ & $15,39 \%$ \\
\hline \multicolumn{4}{|l|}{ 4.2. Hampering factors relating to the migration process } \\
\hline 4.2.1. First cultural shock: differences in habits & 5 & $3 / 13$ & $23,08 \%$ \\
\hline 4.2.2. Perceiving the differences and situations of discrimination & 12 & $7 / 13$ & $53,85 \%$ \\
\hline \multicolumn{4}{|l|}{ 4.3. Hampering factors relating to the housing } \\
\hline 4.3.1. Difficulties arising from the domestic situation & 2 & $2 / 13$ & $15,39 \%$ \\
\hline 4.3.2. Situation of the family home and having to move & 5 & $4 / 13$ & $30,77 \%$ \\
\hline 4.3.3. Difference between urban and rural environments & 3 & $3 / 13$ & $23,08 \%$ \\
\hline \multicolumn{4}{|l|}{ 5. SCHOOL AS A KEY ELEMENT FOR SCHOOL SUCCES } \\
\hline \multicolumn{4}{|l|}{ 5.1. The positive impact of a good adaptation process } \\
\hline 5.1.1. The importance of a prior knowledge of some people & 1 & $1 / 13$ & $7,69 \%$ \\
\hline 5.1.2. Existence of other immigrant students in the school & 4 & $4 / 13$ & $30,77 \%$ \\
\hline 5.1.3. Overcoming changing the educational level and the school & 6 & $4 / 13$ & $30,77 \%$ \\
\hline 5.1.4. Overcoming the lack of familiarity with spaces and people & 14 & $6 / 13$ & $46,15 \%$ \\
\hline \multicolumn{4}{|l|}{ 5.2. The subjects of the school: the teachers } \\
\hline 5.2.1. High expectations of teachers & 15 & $7 / 13$ & $53,85 \%$ \\
\hline 5.2.2. The characteristics of a good teacher & 34 & $11 / 13$ & $84,62 \%$ \\
\hline \multicolumn{4}{|l|}{ 5.2.3. Teachers behaviour: } \\
\hline 5.2.3.1. The positive reinforcement by teachers & 5 & $3 / 13$ & $23,08 \%$ \\
\hline 5.2.3.2. The intervention of teachers beyond the academic context & 6 & $5 / 13$ & $38,46 \%$ \\
\hline 5.2.3.3. The interest to know the culture of the student & 1 & $1 / 13$ & $7,69 \%$ \\
\hline 5.3. The subjects of the school: the management team & 5 & $4 / 13$ & $30,77 \%$ \\
\hline \multicolumn{4}{|l|}{ 5.4. School organizational solutions } \\
\hline 5.4.1. The newcomers programs & 15 & $3 / 13$ & $23,08 \%$ \\
\hline 5.4.2. The inclusive methodologies & 2 & $1 / 13$ & $7,69 \%$ \\
\hline 5.4.3. The review sessions & 4 & $2 / 13$ & $15,39 \%$ \\
\hline 5.4.4. The heterogeneous grouping & 4 & $4 / 13$ & $30,77 \%$ \\
\hline
\end{tabular}




\begin{tabular}{|c|c|c|c|}
\hline \multirow[b]{2}{*}{ 6. ELS ENTORNOS DE EDUCACIÓN NO PARA EL ÉXITO EDUCATIVO } & \multirow{2}{*}{$\begin{array}{c}\begin{array}{c}\text { Frecuencia } \\
\text { total }\end{array} \\
1\end{array}$} & \multicolumn{2}{|c|}{$\begin{array}{l}\text { Número (y \%) de relatos } \\
\text { en las que aparece }\end{array}$} \\
\hline & & $1 / 13$ & $7,69 \%$ \\
\hline \multicolumn{4}{|l|}{ 7. EL ENTORNO PARA EL ÉXITO EDUCATIVO } \\
\hline 7.1. Ayuda de las personas autóctonas del barrio o municipio & 2 & $2 / 13$ & $15,39 \%$ \\
\hline 7.2. Ayuda de los vecinos inmigrantes & 1 & $1 / 13$ & $7,69 \%$ \\
\hline 7.3. Ayuda de los vecinos autóctonos inmigrantes dentro de España & 1 & 1/13 & $7,69 \%$ \\
\hline 7.4. Soporte del entorno del país de origen & 2 & $2 / 13$ & $15,39 \%$ \\
\hline \multicolumn{4}{|l|}{ 7.5. Suporte de las amistades } \\
\hline 7.5.1. Ayuda académica & 5 & $3 / 13$ & $2,3 \%$ \\
\hline 7.5.2. Ayuda económica & 3 & 1/13 & $7,69 \%$ \\
\hline \multicolumn{4}{|l|}{ 7.5.3. Acompañamiento, tutela,... } \\
\hline \multicolumn{4}{|c|}{ 8. OTROS ELEMENTOS FAVORECEDORES DEL SISTEMA EDUCATIVO Y SOCIAL } \\
\hline 8.1. Becas: ayudas económicas del sistema & 10 & $5 / 13$ & $38,46 \%$ \\
\hline 8.2. Figura del asistente social para la integración de les familia & 2 & $1 / 13$ & $7,69 \%$ \\
\hline 9. OTROS ELEMENTOS DIFICULTADORES & 19 & $5 / 13$ & $38,46 \%$ \\
\hline 10. LA LENGUA COMO ELEMENTO CLAVE: el catalán & 36 & $10 / 13$ & $76,92 \%$ \\
\hline
\end{tabular}

\section{Resultado}

Tal y como podemos observar en la tabla 3, en los relatos de vida las competencias más citadas como ejes clave han sido la capacidad de comunicarse, las competencias sociales y cívicas, el sentido de la iniciativa y autonomía, y finalmente, lo que más aparece en todos los relatos ha sido la cultura del esfuerzo y responsabilidad (18 veces y aparece en 9 de los 13 relatos de vida), dentro de las competencias emocionales.

"Miforma de ser, el hecho de auto realizarme, fueron aspectos que me ayudaron a estudiar y a llegar a la universidad" (relato de K.A.A.).

"Desde pequeña he aprendido que si quiero alguna cosa tengo que espabilarme yo sola para conseguirla" (relato de L.H.).

En relación con los elementos y acciones personales que pueden influir en el éxito, con casi el mismo número de referencias, aparece el género percibido como elemento de dificultad y, como potenciadores del éxito, los sentimientos relacionados con el mismo como la auto percepción positiva ligada al sentido de la iniciativa y la autonomía, a la expectativas de futuro (concretamente al hecho de ir a la universidad), y a la capacidad de alejarse de ciertas 'amistades', aspecto que aparece cuando los estudiantes hacen referencia a la necesidad de rom-

\section{Results}

Thus, it can be observed in table 3, that in the life stories the competencies most often cited as key factors for academic success have been to ability to communicate, the social and civic competencies, the sense of initiative and autonomy, and finally, what has been displayed in all the stories has been the dedication and personal effort ( 18 times and appears in 9 of the 13 life stories).

"My way of being and the fact of auto fulfil myself were aspects that helped me to study and go to college" (life story of K.A.A.).

"Since I was a child I have learned that if I want something I have to fight for it alone to get it" (life story of L.H.).

Relating to the elements and actions that can influence on school success, with almost the same number of references, gender appears as an element of difficulty. As enhancers of success the feelings associated with an auto positive perception linked with the sense of initiative and autonomy, the future expectations (and specifically the fact of wanting to go to college), and the ability to get away from certain 'friends' (something that appears when students refer to the need to break with ghettos created within the school to be able to

[ 276 ] ELENA CANO GARCIIA Y MAITE FERNÁNDEZ FERRER 


\begin{tabular}{|c|c|c|c|}
\hline & Frequency & \multicolumn{2}{|c|}{ Numerical analysis } \\
\hline 6. THE EDUCATIONAL ENVIRONMENT NOT FOR THE SCHOOL SUCCES & 1 & $1 / 13$ & $7,69 \%$ \\
\hline \multicolumn{4}{|l|}{ 7. THE EDUCATIONAL ENVIRONMENT FOR THE SCHOOL SUCCES } \\
\hline 7.1. Help from native people of the district & 2 & $2 / 13$ & $15,39 \%$ \\
\hline 7.2. Help from the immigrant neighbours & 1 & $1 / 13$ & $7,69 \%$ \\
\hline 7.3. Help from the immigrants native neighbours in Spain & 1 & $1 / 13$ & $7,69 \%$ \\
\hline 7.4. Support from the home country & 2 & $2 / 13$ & $15,39 \%$ \\
\hline \multicolumn{4}{|l|}{ 7.5. Support from the friends } \\
\hline 7.5.1. Academic assistance & 5 & $3 / 13$ & $2,3 \%$ \\
\hline 7.5.2. Financial assistance & 3 & $1 / 13$ & $7,69 \%$ \\
\hline \multicolumn{4}{|l|}{ 7.5.3. Support and care } \\
\hline \multicolumn{4}{|l|}{ 8. ANOTHER KEY ELEMENTS OF THE SOCIAL AND EDUCATION SYSTEM } \\
\hline 8.1. Scholarships: financial support programms & 10 & $5 / 13$ & $38,46 \%$ \\
\hline 8.2. The figure of the social worker & 2 & $1 / 13$ & $7,69 \%$ \\
\hline 9. ANOTHER HAMPERING ELEMENTS & 19 & $5 / 13$ & $38,46 \%$ \\
\hline 10. THE LANGUAGE: CATALAN & 36 & $10 / 13$ & $76,92 \%$ \\
\hline
\end{tabular}

per con los guetos creados dentro de la escuela para poder romper con los estereotipos culturales y avanzar académicamente.

[En relación al género] "Cuando llegué en el instituto tenía muy claro que quería seguir estudiando mientras que mis padres no lo tenían demasiado claro, decían que ya sabía leer y escribir y que esto era suficiente" (relato de L.H.).

En relación con los elementos favorecedores de la inclusión social destacamos los relativos a la familia ya que ésta aparece como agente clave en el desarrollo personal del estudiante y en el éxito. De esta manera, las expectativas de la familia (15), las acciones de la familia para dar soporte a sus hijos e hijas (11) (por ejemplo, ayudando en la realización de los deberes, asistiendo a las reuniones, animando en sus estudios, insistiendo en que sean responsables, etc.), y la valoración positiva que otorga la familia a la importancia de la educación como mecanismo de promoción social (16), son los elementos más apreciados y citados por parte de los informantes de los relato de vida:

“En mi opinión, el hecho de que mis padres valoraran el éxito académico, que nos reforzaran verbalmente cuando sacábamos buenas notas y que fueran muy estrictos con nuestros horarios y nuestro tiempo libre son factores clave para que nosotros siguiéramos estudiando" (relato de K.S.). break cultural stereotypes and advance academically) were perceived by immigrant students.

[Relating to the gender] "When I got into high school for me it was very clear that I wanted to continue studying while my parents were not too clear. They said I already knew how to read and write and that this was enough" (life story of L.H.).

Regarding the key factors for social inclusion it is highlighted those elements relating to the family and it appears as a key agent in the student's personal development and success. Thus, family expectations (15), the actions of the family to support the education of their children (11) (for example, helping with homework and/or class work or Insisting on the importance of being responsible), and the positive value that gives the family on the importance of education as a means of social promotion (16), are the most prized elements cited by informants through life stories:

"In my opinion, the fact that my parents valued academic success, that they reinforced us verbally when we had good grades and that they were very strict with our schedules and our free time, were key factors for us to follow studying" (life story of K.S.). 
Contrariamente, respecto a los elementos de dificultad de la inclusión social, los elementos relativos a la familia son los que vuelven a aparecer con más fuerza, concretamente los recursos económicos limitados de la misma (11) y la religión familiar (10), es decir, la influencia de algunas tradiciones y costumbres que son traspasadas de padres y madres a hijos e hijas. Finalmente, con menos frecuencia, otro elemento referido a esta categoría ha sido el relativo a la vivienda referido al estado o situación de la misma y al hecho de tener que desplazarse para llegar al centro educativo $(30,70 \%)$.

"Yo en Marruecos tenía muy claro que como mucho llegaría a sexto porque en el pueblo hay solo primaria y después ya me quedaría en casa hasta que viniera algún pretendiente a pedirme la mano" (relato de L.H.).

Por otro lado, si nos referimos a la escuela como elemento central para el éxito escolar, se hace necesario destacar dentro del impacto positivo de un proceso de adaptación el hecho de superar el desconocimiento de los espacios y de las personas, aspecto que provoca una gran desorientación entre los estudiantes (14) y por lo tanto, que va acompañado de sentimientos negativos. Dentro del centro escolar aquello que destaca más y sobre lo que se ha hecho más referencia es la figura del docente. Cabe destacar que las altas expectativas del profesorado son valoradas muy positivamente para adquirir el éxito por parte de los estudiantes:

\footnotetext{
"Un tutor le dijo una vez a mi madre que podía estudiar lo que quisiera ya que tenía capacidad para hacerlo" (relato de OO).

"Ha sido un constante feedback: Ellos creían en mí y yo creía en ellos" (relato de SH).
}

Además, las características del buen profesor definidas en los mismos relatos de vida aparecen un gran número de veces, concretamente 34 , y en un $84,62 \%$ de los relatos.

“(...) Los profesores siempre me han ayudado y preguntado cosas sobre nuestra cultura" (relato de SH).

Finalmente, con relación al centro escolar como factor para el impulso del éxito, la solución organi-
Conversely, regarding the hampering factors for social inclusion, the elements concerning the family are the ones that appear again more strongly. These were concretely the limited financial resources of the family (11) and the family religion (10) with the influence of certain traditions and customs that are transferred from parents to children. Finally, less frequently, another element referred to this category has been the hampering factors relating to the housing, concretely, its situation and the fact of having to move far away to get to school every day (30.70\%).

"In Morocco I was very conscious that as much I would only reach sixth grade because in my village there was only elementary school. After that I knew I would stay home until a man came to propose and marry me" (life story of L.H.).

On the other hand, if we refer to school as a key element for school success, it is necessary to emphasize within the positive impact of an adaptation process the fact of overcoming the lack of familiarity with spaces and people, something that causes great disorientation among students (14) and therefore that it is accompanied by negative feelings. Within the school that which stands out the most and hat has been more referenced is the figure of the teacher. The high expectations of teachers are highly appreciated to acquire success by students:

\footnotetext{
"A teacher once told my mother I could study whatever I wanted as I had capacity to do so" (life story of $\mathrm{OO})$.

"It has been a constant feedback: They believed in me and I believed in them" (life story of SH).
}

In addition, the characteristics of a good teacher defined in the life stories appeared a large number of times, namely 34 , and in $84.62 \%$ of the stories. Finally, still concerning the school context as a key factor for success, their organizational solutions and specifically, the newcomers programs have been also highlighted as an important element for students (15), even sometimes it was valued positively and another times negatively.

[ 278 ] ELENA CANO GARCÍA Y MAITE FERNÁNDEZ FERRER 
zativa de la escuela referida a las aulas de acogida también han sido un elemento importante para los estudiantes (15), aunque a veces valorada positivamente y otras de forma negativa.

Por último, destacamos el apoyo de las amistades, concretamente en la función de acompañamiento y tutela $(38,46 \%)$ y ciertos elementos favorecedores del sistema educativo y social como son las becas $y / 0$ las ayudas económicas del sistema (38,46\%).

\footnotetext{
“También recibí ayuda por parte de algunos compañeros que se ofrecían voluntarios para ayudarme, para aprenderme sus nombres, para jugar con ellos en el patio..." (relato de L.H.).

“También es muy importante reconocer el valor que tienen las becas del estado, puesto que sin ellas mucha gente no podría estudiar (como es mi caso)" (relato de S.H.).
}

Finalmente, la lengua como elemento clave, concretamente el catalán, ha sido uno de los ejes que ha aparecido en mayor frecuencia en todo el conjunto de los relatos (36) y además, en casi todos los escritos $(76,92 \%)$.

\section{Discusión y conclusiones}

\section{Respecto a las competencias clave}

Las competencias que los estudiantes aseguran haber desarrollado más a lo largo de sus estudios y que consideran que les han ayudado a llegar a la universidad han sido dos principalmente: la competencia de autonomía e iniciativa personal (que es una de las competencias clave) y la responsabilidad y auto exigencia (que podría vincularse a la competencia de aprender a aprender). Dentro de esta última, la cultura del esfuerzo ha sido la más valorada por los participantes, entendiendo ésta como el propio trabajo autónomo de los estudiantes para llegar a conseguir sus objetivos a pesar de los obstáculos, así como la auto exigencia vinculada a la motivación intrínseca, a la resiliencia y a la auto eficacia (Zimmerman, 2008).

Además es necesario destacar que la capacidad de comunicarse (nuevamente una de las competencias básicas) ha sido muy citada y valorada por los participantes de la investigación, ya que ha estado muy ligada a la creencia de la lengua
“(..) Teachers always helped me and asked me things about my culture" (life story of SH).

Finally, in the life stories it is highlighted on one hand the support from the friends, particularly in their role of support and care (38.46\%). Secondly, another key element of the social and education system has been the scholarships and the financial support programs (38.46\%). And thirdly, another hampering elements not mentioned above (38.46\%).

"I also received help from some fellow colleagues who offered to help me to learn their names, to play with them in the yard..." (life story of L.H.).

"It is also important to recognize the value of these scholarships from the state since without them many people would not even consider to study (like me)" (life story of S.H.).

In conclusion, the language has been as a key element, concretely the Catalan language, and the factor that has appeared more frequently in the life stories (36) and also in almost all of them (76.92\%).

\section{Discussion and conclusions}

\section{Relating to key competencies}

The competencies that students say they have developed the most throughout their studies and that have helped them to get to college are mainly two: sense of initiative and autonomy and self efficacy and feeling capable. Within this last one, dedication and personal effort has been the most valued by participants, defined as the own work of students to get to achieve their goals despite of the obstacles, as well as competencies related to intrinsic motivation, resilience and self-efficacy (Zimmerman, 2008).

In addition, it should be noted that the ability to communicate has been widely cited and valued by the research participants as it has been closely linked to the belief of the language as a key element in achieving the goals (in this particular case, the Catalan language) (Serra \& Palaudàries, 2010). Although it has been an element of difficulty in most cases, having acquired the knowledge of this 
como elemento clave para alcanzar los objetivos, en este caso en concreto, la lengua catalana (Serra y Palaudàrias, 2010). Por último, aunque ha sido percibido como un elemento de dificultad en la mayoría de los casos, cuando se ha adquirido el conocimiento de la lengua, tanto hablado como escrito, éste se valora como un hecho que les ha ayudado no sólo a un mayor rendimiento académico sino también a una mejor integración en los espacios de ocio con sus compañeros. $Y$ con relación con este último aspecto, es necesario poner énfasis en las competencias social y cívica o ciudadana (la última de las competencias clave), es decir, la importancia de la capacidad de establecer contacto con los otros, independientemente de las barreras sociales, económicas y culturales.

\section{Respecto al éxito educativo}

El éxito educativo va ligado totalmente al abandono escolar y, por lo tanto, a la falta de habilidades para la inserción laboral con éxito. Los informantes de la investigación aseguran que hay un conjunto de factores que han influido en su éxito escolar. Estos factores y variables serian principalmente el apoyo de la familia (Suárez Orozco \& Suárez Orozco, 1995; Marí-Klose, Marí-Klose, Vaquera \& Cunningham, 2010; Enriquez, 2011; Crosnoe \& López, 2011) así como la auto imagen positiva que tienen sobre ellos mismos y sus propias expectativas (Yeung \& Mclnerney, 2005), dos aspectos que además van relacionados entre sí porque aparecen conjuntamente en muchas de las respuestas. Además aseguran que los elementos y acciones personales que han sido los sentimientos relacionados con el éxito como pueden ser esta auto percepción positiva (su capacidad de decisión, de mentalidad abierta, etc.) y las altas expectativas de futuro, es decir, el objetivo seguro y claro de llegar a la universidad.

Dentro de la institución educativa, el elemento principal que ha sido decisivo para los estudiantes inmigrantes ha sido el profesorado. La mayoría de los participantes de la investigación aluden a las características de algún buen profesor y a las altas expectativas del docente respeto a la posibilidad de ir a la universidad como elemento de gran ayuda para su desarrollo social y académico, destacando language, both spoken and written, has helped them to better integrate with their peers. In respect to this last point, it is necessary to emphasize the social and civic competencies. The importance of establishing contact with the colleagues has helped the students to relate to others and thus to decrease situations arising from discrimination among peers feeling equal in front of the others and getting the same goals regardless of social, economic and cultural barriers.

\section{Relating to school success}

The school success is linked entirely to dropout and therefore, to the lack of competencies for a successful employment. The research informants say there are a number of factors that have influenced their academic success. These factors and variables would primarily be the support of their family (Suárez Orozco, 1995; Marí-Klose, Marí-Klose, Cowgirl \& Cunningham, 2010; Enriquez, 2011; Crosnoe and Lopez, 2011) and the positive self-image they have on themselves and their own expectations (Yeung \& Mclnerney, 2005), both of which are interrelated also as they appear together in many of the answers. Immigrant college students highlight their positive self-perception (their decision-making or the fact of being open-minded) and the importance of their high expectations for the future referring to the confident and clear goal to get to college.

Inside the education institution the main element that has been decisive for immigrant students has been the faculty members. Most research participants refer to the characteristics of a good teacher and their high expectations on them to go to college as a key element for their social and academic development. Specifically informants highlight teachers' monitoring and their close control of each student. Therefore, it is been found a positive value of the teachers, not from a disciplinary point of view but beyond the instruction as an active member in the accompaniment of the young. Moreover, within the school, a friendly school climate or an affective environment (Murillo, 2005) also helps to a high a positive student performance. 
concretamente el seguimiento y control cercano de cada estudiante por parte de estos profesores. Por lo tanto, se produce una valoración positiva del profesor o profesora, no desde el punto de vista disciplinar sino más allá de la instrucción, es decir, como miembro activo en el acompañamiento del joven. Además, dentro del centro educativo, el clima escolar agradable y/o el ambiente afectivo (Murillo, 2005) también contribuyen a un mejor rendimiento del estudiante.

\section{Respecto a la inclusión social}

Los estudiantes hijos/as de inmigrantes destacan los elementos relativos a la familia y el más citado ha sido la valoración que ésta otorga a la educación, es decir, al hecho de creer en ella como mecanismo de promoción social, así como las expectativas que tienen en relación con la misma. Dentro de la familia se hace necesario destacar que el elemento más citado ha sido la figura de la madre (Aldous, 2006; Calero et al., 2006; Marí-Klose, Marí-Klose, Vaquera \& Cunningham, 2010), valorada muy positivamente como punto clave para el apoyo y acompañamiento a lo largo de la infancia y la adolescencia (así como la figura de los hermanos y hermanas en algunos casos), mientras que la figura del padre se muestra en pocos casos e incluso en alguna ocasión se alude a él negativamente.

Los elementos favorecedores relativos al proceso de inmigración han sido el hecho de entender el aspecto de vivir con otras culturas como elemento enriquecedor (Arnáiz \& De Haro, 2004) y, por lo tanto, ver el proceso de inclusión de manera positiva y optimista. Contrariamente, los elementos de dificultad del proceso de inclusión social y que, por lo tanto, entorpecen el éxito educativo han sido principalmente los recursos económicos limitados de la familia y la percepción que han tenido los estudiantes de sus diferencias en las situaciones de discriminación. Además es necesario destacar la religión familiar (Besalú \& Vila, 2007) como un punto importante en los procesos de integración social de los niños, niñas y jóvenes inmigrantes aunque parece mucho más relevante el nivel de formación de los padres y sus expectativas.
Thus, as it has been seen, we find a set of generic factors and elements related to the context (socioeconomic status, gender, student performance), in which professionals can intervene.

\section{Relating to social inclusion}

Social inclusion is a new way of understanding education placing first the inequality and exclusion that during the investigation has appeared in many of the cases analysed. The students' children of immigrant families include elements relating to the family and the most often cited was their parents positive value of education and the fact of believing in it as a key for social promotion. Within the family is necessary to emphasize that the most appeared element has been the mother figure (Aldous, 2006; Calero et al, 2006; Marí-Klose, Marí-Klose, Cowgirl \& Cunningham, 2010), highly appreciated as a key point for the support and assistance throughout childhood and adolescence (and the figure of the brothers and sisters in some cases). While the father figure, shown in these few cases, has been referenced as an authoritarian, with little belief in education as an important factor for change.

The predisposing factors relating to the immigration process have been the positive value of $\mathrm{li}$ ving with other cultures as an enriching element (Arnaiz \& De Haro 2004). Conversely, the elements of difficulty of the process for social inclusion have mainly been by far the limited economic resources of the family and the perception of being different that students have had in the situations of discrimination. In addition it should be noted the family religion (Besalu \& Vila, 2007) as an important step in the process of social integration of children and young immigrants although it seems much more relevant the educational level of their parents and their future expectations point. 


\section{REFERENCIAS BIBLIOGRÁFICAS / REFERENCES}

Aja, E. D., Essomba, P., Formariz, M.A., \& Girona, A. (2000). Educació $i$ immigració. Els reptes educatius de la diversitat cultural i l'exclusió social. Barcelona: Editorial Mediterrània

Alarcón, A. (2007). Joves d'origen immigrant a Catalunya. Necessitats $i$ demandes. Una aproximació sociológica. Barcelona: Fundació Jaume Bofill.

Aldous, J. (2006). Family, Ethnicity and Immigrant Youths' Educational Achievements. Journal of Family Issues, 27 (12), 1633-1667.

Alegre Canosa, M. A. (2005). Educació i immigració: l'acollida als centres educatius. Barcelona: Editorial Mediterrània.

Alegre, M. A., Benito, R., \& González, S. (2006). Immigrants als instituts. L'acollida vista pels seus protagonistes. Barcelona: Editorial Mediterrània.

Aparicio, R., \& Tornos, A. (2006). Hijos de inmigrantes que se hacen adultos: Marroquíes, dominicanos, peruanos. Documentos de Observatorio Permanente de la Inmigración, 8.

Arnaiz, P., \& De Haro, R. (2004). Ciudadanía e interculturalidad: claves para la educación del siglo XXI. Educatio Siglo XXI, Retrieved from http://revistas.um.es/educatio/article/view/97

Besalú, X., \& Vila, I. (2007). La buena educación: libertad e igualdad en la escuela del siglo XXI. Barcelona: La Catarata.

Calero, J. (Coord.) (2006). El rendimiento educativo del alumnado inmigrante analizado a través de PISA 2006. Madrid: Estudios CREADE.

CIDE-MEC (2005). La atención al alumnado inmigrante en el sistema educativo en España. Madrid: CIDEMEC.

Comisión Europea (2010). Europa 2020. Estrategia para un crecimiento Inteligente, Sostenible e Integrador. Bruselas: COM.

Crosnoe, R., \& López, R.N. (2011). K-12 Educational Outcomes of Immigrant Youth. Future of children, 21 (1), 129152.

Enriquez, L.E. (2011). Because We Feel the Pressure and We Also Feel the Support": Examining the Educational Success of Undocumented Immigrant Latina/o Students. Harvard Educational Review, 81 (3), 476499.

Escamilla, A, (2008). Concepto y funciones de las competencias básicas. Bases para su desarrollo en los centros. Librosvivos. Net

Estellés, P. (Coord.) (2012). El fracàs escolar a Catalunya. Informe FEDAIA. Barcelona: FEDAIA.

Ferrer, F. (Dir.) (2009). Equitat, excel.lència i eficència educativa a Catalunya. Una anàlisi comparada. Barcelona: Editorial Mediterrània/ Fundació Bofill.

Generalitat de Catalunya (2005). Pla de Ciutadania i immigració 2005 - 2008. Barcelona: Generalitat de Catalunya.

Generalitat de Catalunya (2010). Pla de Ciutadania i Immigració 2009 - 2012. Barcelona: Generalitat de Catalunya.

Huguet, A. \& Navarro, J. (2006). Inmigración y resultados escolares: lo que dicen las investigaciones. Cultura y Educación, 18, 117-126.

Instituto de Evaluación (2010). PISA 2009. Informe español. Madrid: Ministerio de Educación.

Mari-Klose, P., Mari-Klose, M., Vaquera, E. \& Cunningham, S.A. (2010). Infancia y futuro. Nuevas realidades, nuevos retos. Colección Estudios Sociales, 30.

Monereo, C. (Coord.) (2009). PISA como excusa: repensar la evaluación para cambiar la enseñanza. Barcelona: Graó.

Murillo, F. J. (2005). La investigación sobre eficacia escolar. Barcelona: Octaedro.

Navarrete, L. (2007). Jóvenes y Fracaso escolar en España. Madrid: Instituto de Juventud (INJUVE).

OECD (1998). Overcoming Failure at School. Paris: OECD.

[ 282 ] ELENA CANO GARCÍA Y MAITE FERNÁNDEZ FERRER

SIPS - PEDAGOGIA SOCIAL. REVISTA INTERUNIVERSITARIA [1139-1723 (2015) 26, 263-284] TERCERA ÉPOCA 
OECD (2010). PISA 2009 Results: Overcoming social background - equity in learning opportunities and outcomes (volume II). Paris: OECD. doi: 10.1787/9789264091504-en

Orfield, G. (2001). Schools more separate. Consequences of a Decade of resegregation. Cambridge (MA): Harvard Civil Rights Project.

Oseguera, L., Conchas, G.Q., \& Mosqueda, E. (2011). Beyond Family and Ethnic Culture: Understanding the Preconditions for the Potencial Realization of Social Capital. Youth \& Society, 43 (3), 1136-1166.

Serra, C., \& Palaudàrias, J.M. (2010). Continuar o abandonar. L'alumnat estranger a l'educació secundària. Barcelona: Fundació Jaume Bofill.

Suárez Orozco, C. \& Suárez Orozco, M. (1995). Transformations: immigration, family, life and achievement motivation among Latino adolescents. Stanford: Stanford University Press.

Truñó, M. (2012). Infància a Catalunya. Barcelona: UNICEF Comitè Catalunya.

Yeung, A.S. \& Mclnerney, D.M. (2005). Student's School Motivation and Aspiration Over High School Years. Educational Psychology, 25 (5), 537-554.

Zimmerman, B. J. (2008). Investigating Self-Regulation and Motivation: Historical Background, Methodological Developments, and Future Prospects. American Educational Research Journal, 45 (1), 166-183.

\section{CÓMO CITAR ESTE ARTÍCULO / HOW TO CITE THE ARTICLE}

Cano García, E. \& Fernández Ferrer, M. (2015). La visión de los estudiantes de origen extranjero sobre factores y competencias clave para el acceso a la Universidad. Pedagogía Social. Revista Interuniversitaria, 26 263-284. DOI:10.7179/PSRI_2015.26.10

Fecha de recepción del artículo / received date: 28.VI.2014

Fecha de revisión del artículo / reviewed date: 1.VII.2O14

Fecha de aceptación final / accepted date: 25.XI.2014

\section{DIRECCIÓN DE LAS AUTORAS/ AUTHORS' ADDRESS}

Elena Cano García. Dirección de correo/e-mail: Passeig de la Vall d'Hebron, 171. 08035 BARCELONA. Dirección de correo/e-mail: ecano@ub.edu

Maite Fernández Ferrer. Mundet, Campus Mundet, Llevant, PI. 2a. PG.VALL D'HEBRON, 171. 08035 BARCELONA. Dirección de correo/e-mail: maite.fernandez@ub.edu

\section{PERFIL ACADÉMICO / ACADEMIC PROFILE}

Elena Cano García. Profesora titular del Departamento de Didáctica y Organización Educativa de la Universidad de Barcelona. Ha investigado sobre calidad de la educación (Evaluación de la Calidad Educativa, La Muralla) y evaluación educativa (Aprobar o aprender, Transmedia XXI). En los últimos años ha trabajado en especial sobre la evaluación de competencias en la educación superior (Buenas prácticas para la evaluación de competencias, Laertes), tema sobre el que ha dirigido varios proyectos y acerca del cual coordina un proyecto $1+D$ actualmente.

Maite Fernández Ferrer. Licenciada en Pedagogía por la Universitat de Barcelona y Máster en Estudios Internacionales y en Intervenciones Sociales y Educativas por la misma universidad. Miembro del Grupo de Investigación Consolidado Laboratorio de Medios Interactivos (GRC-LMI). Ha formado parte del equipo de distintas investigaciones sobre evaluación y competencias en el ámbito universitario y des de 2011 ha participado en diferentes encuentros científicos sobre la temática, tanto nacionales como internacionales, de 
los que han derivado varias publicaciones. Entre su experiencia académica internacional se encuentra su estada de seis meses en la School of Education de la University of Cape Town (Sudáfrica), participando en un estudio sobre la calidad de la educación secundaria en los 'townships' del país. Actualmente trabaja como Personal Investigador Novel (FI-DGR 2014) en la Universidad de Barcelona donde está realizando su tesis doctoral sobre los Cursos Masivos Abiertos Online y su evaluación. 